\title{
SEARCHING A SOCIAL NETWORKING SITE USING NATURAL LANGUAGE PROCESSING TECHNIQUE
}

\section{Aamir Junaid Ahmad ${ }^{1}$ and Sabina Priyadarshinin ${ }^{2}$}

\begin{abstract}
${ }^{1}$ Department of Computer Science \& Engineering, Maulana Azad College of Engineering \& Technology, Patna, India ${ }^{2}$ Department of Information Technology Birla Institute of Technology, Mesra, India
\end{abstract}

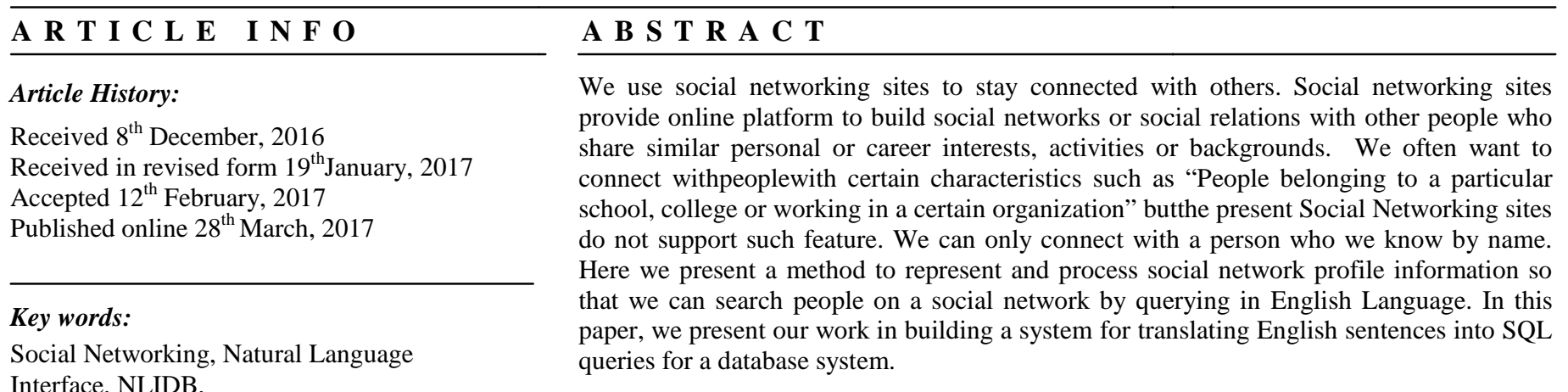

Copyright $@ 2017$ Aamir Junaid Ahmad and Sabina Priyadarshini. This is an open access article distributed under the Creative Commons Attribution License, which permits unrestricted use, distribution, and reproduction in any medium, provided the original work is properly cited.

\section{INTRODUCTION}

Social Networking is an easy way for people to have a social connection with other people with similar interests[1]. These sites serve communication purposes among special interest groups[2], but they do not have a proper searching option where we can look for people or groups having certain characteristics.

If we want list of people with the following characteristics

1. Assistant Professors in the Computer Science Department living in Ranchi

2. Professors in Computer Science Department of BIT Mesra

3. System Analysts working at Infosys in Bangaluru city.

The above query in English Language does not give the results as expected.

*Corresponding author: Aamir Junaid Ahmad

Department of Computer Science \& Engineering, Maulana Azad

College of Engineering \& Technology, Patna, India
Query 1. Assistant Professors in the Computer Science department Living in Ranchi.

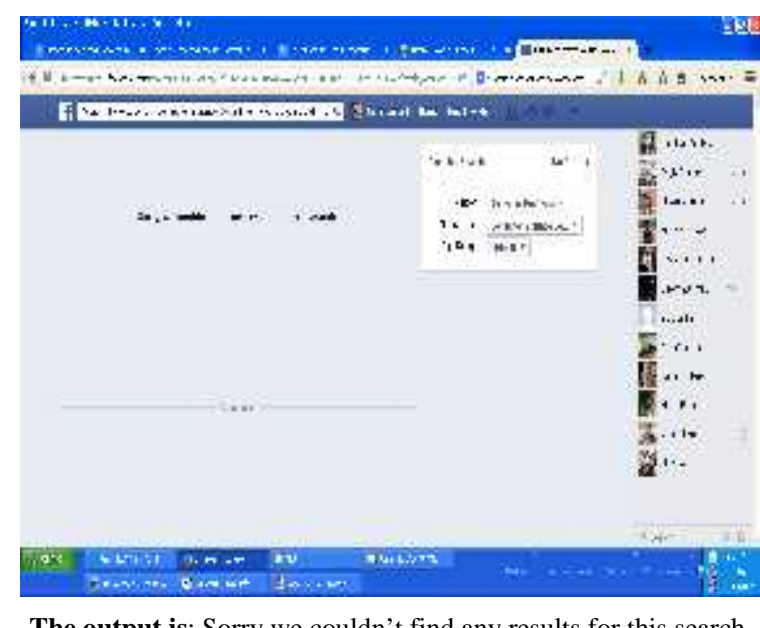

2. Assistant Professors in Computer Science Department who go to BIT Mesra

All of these results are not as expected by the user. In this work we want to add a search option in the social networking sites where we can find people based on their characteristics. 


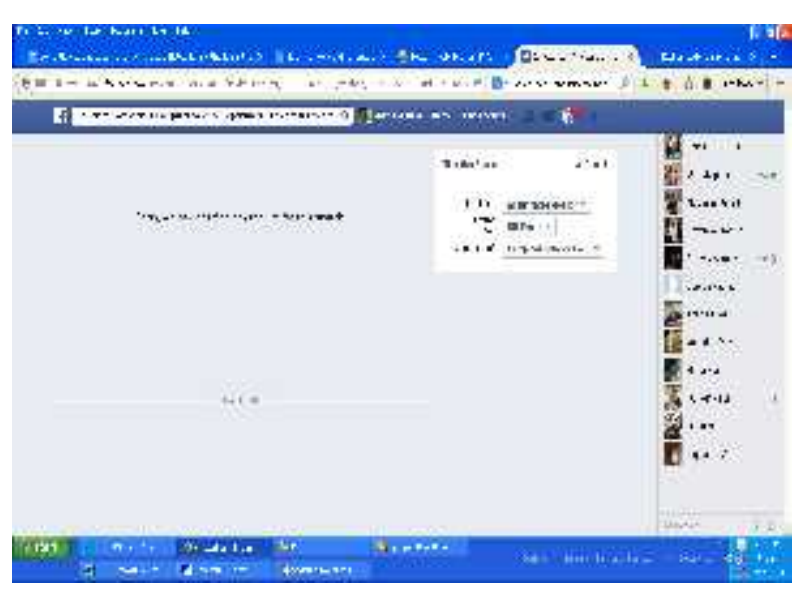

The output is: Sorry we couldn't find any results for this search 3. Assistant Professors in the Computer Science department

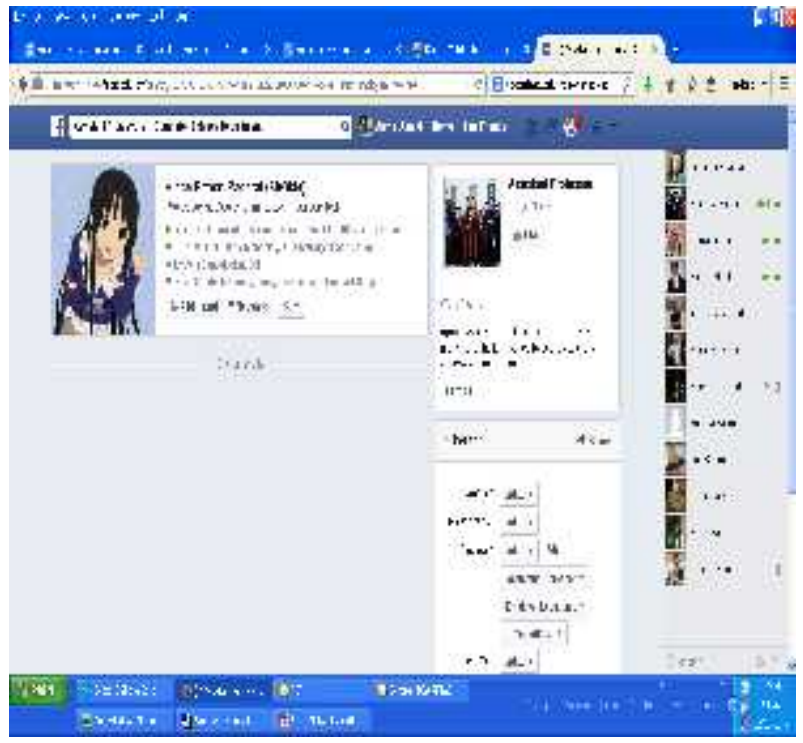

\section{METHODOLOGY}

Natural Language Interfaces to Databases (NLIDBs) [3] are one of the best options for such situations where users look for information from database and formulate nontrivial andinfrequent queries. The translation of a database query expressed in natural languageinvolves the search of relations that link words of the query (nouns, adjectives, etc.) with elements of the database (tables, columns, etc.), which allow to formalize the query in Structured Query Language (SQL). In this work we have used one sample table, Table 1, which contains all those columns which have been mostly used in the query corpus created from potential user.

Following are the few sample queries which can be answered using our system

- Assistant Professor in the department of Computer Science working at BIT Mesra

- All Professors in the department of Electronics working at IIT Patna

- Accountants in Patna

- $\quad$ Students of St. Michaels working in Delhi

- Girls passed out from Notre Dame Academy living in Patna

- Students of Maulana Azad College working in Bengaluru
We use the following list and data structures in our system.

1. List of cities in the world in alphabetical order

2. List of Countries in the world in Alphabetical order

3. List of Designation with synonym of each designation

4. List of Company/Organization

\section{We have created the following algorithm}

1. Accept Natural Language (English Language) query from the user.

2. Remove punctuations (if any)

3. Split the query into tokens

a. Remove prepositions(on, at, it, from total 94)

b. Remove pronouns (you, our)

c. Remove conjunctions (and, but, though)

d. Remove determiners (the, an, this, some)

4. Identify the words in the user's query which are column names in the table, if any, present in the user's query will be used in the where clause/condition in the select statement.

5. Match each word in the list of cities

If foundwe append one condition city='word' in the select statement. Since there are only 4037 cities with population more than 100000 , the sorted list can be searched in $\mathrm{O}(\mathrm{n})$ time.[4] The word is removed from the query.

6. Match each word in the list of countries

If found we append one condition country='word' in the select statement. Since there are only 196 countries the search will be very fast.[5]The word is removed from the query.

7. Match remaining words in the list of Designations

If found we append one condition designation='word' in the select statement. As this table is two dimensional containing Similar designations in each row, the complexity will be $\mathrm{O}\left(\mathrm{n}^{2}\right)$. Since the list is small, for small $\mathrm{n}$ we can consider it as $\mathrm{O}(\mathrm{n})$

The word is removed from the query.

Match remaining words in the list of Colleges[6]

8. If found we append one condition college='word' in the select statement. As this table is two dimensional containing Similar designations in each row, the complexity will be $\mathrm{O}\left(\mathrm{n}^{2}\right)$. Since the list is small, for small $n$ we can consider it as $\mathrm{O}(\mathrm{n})$

Generate the final query and fire it on the database to get the required result which will be displayed to the user.

\section{Implementation}

The above algorithm is implemented using HTML, PHP and MySql. HTML is used to design an interface where the user can enter his query in English Language as shown in the Figure1. This is a simple form with a Textbox and a submit Button. When the user submits his query PHP code is used to process the query on the above mentioned technique/algorithm. Based on the user query equivalent SQL select statement is prepared and fired on the database table, Table 1. The output is shown in Figure 2.PHP and MySqlis used as Facebook was initially written using these tools [7]. PHP and MySql provide good connectivity and is easily available. 
Table 1 UserProfile

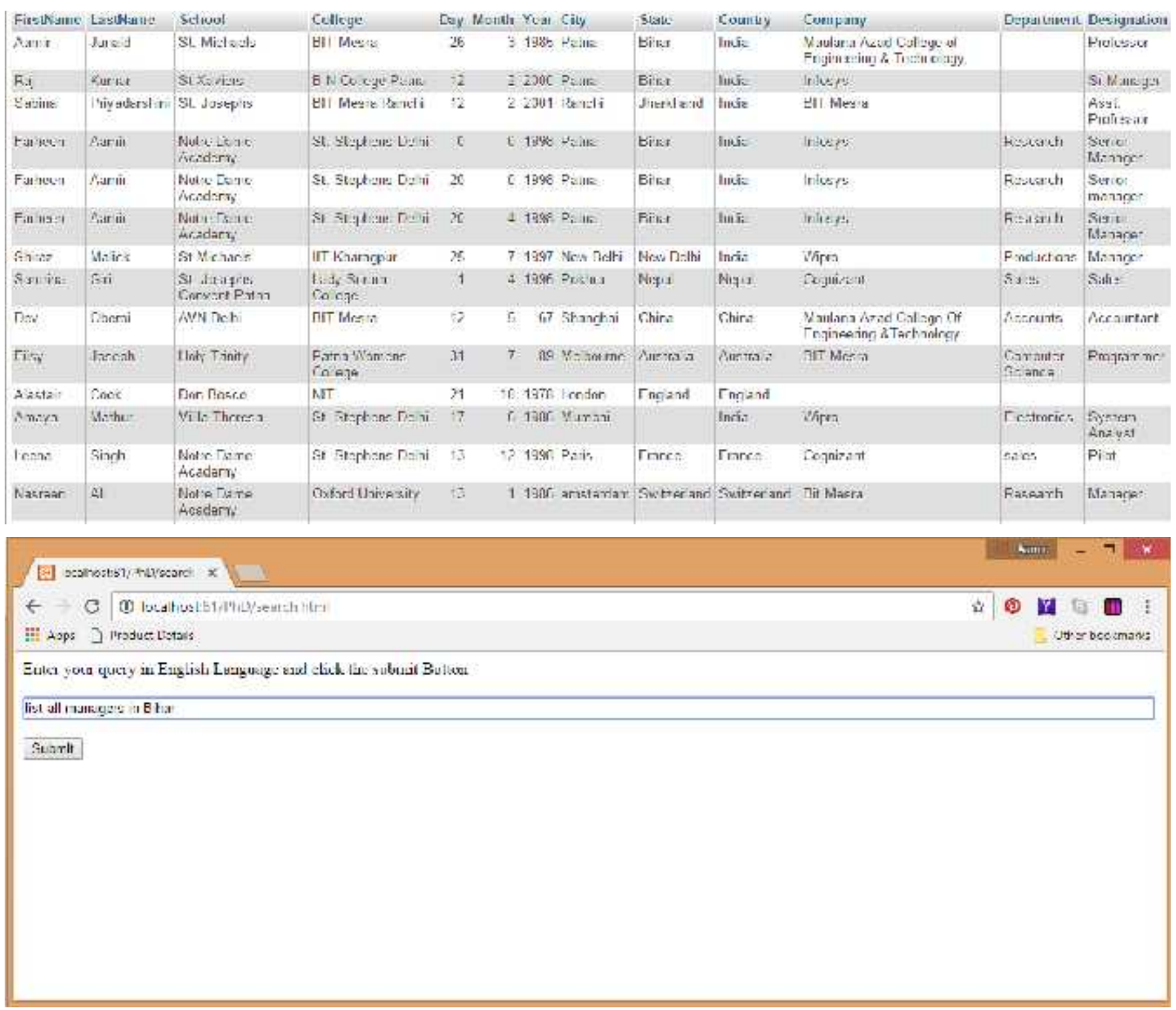

Figure 1 Interface Design in HTML

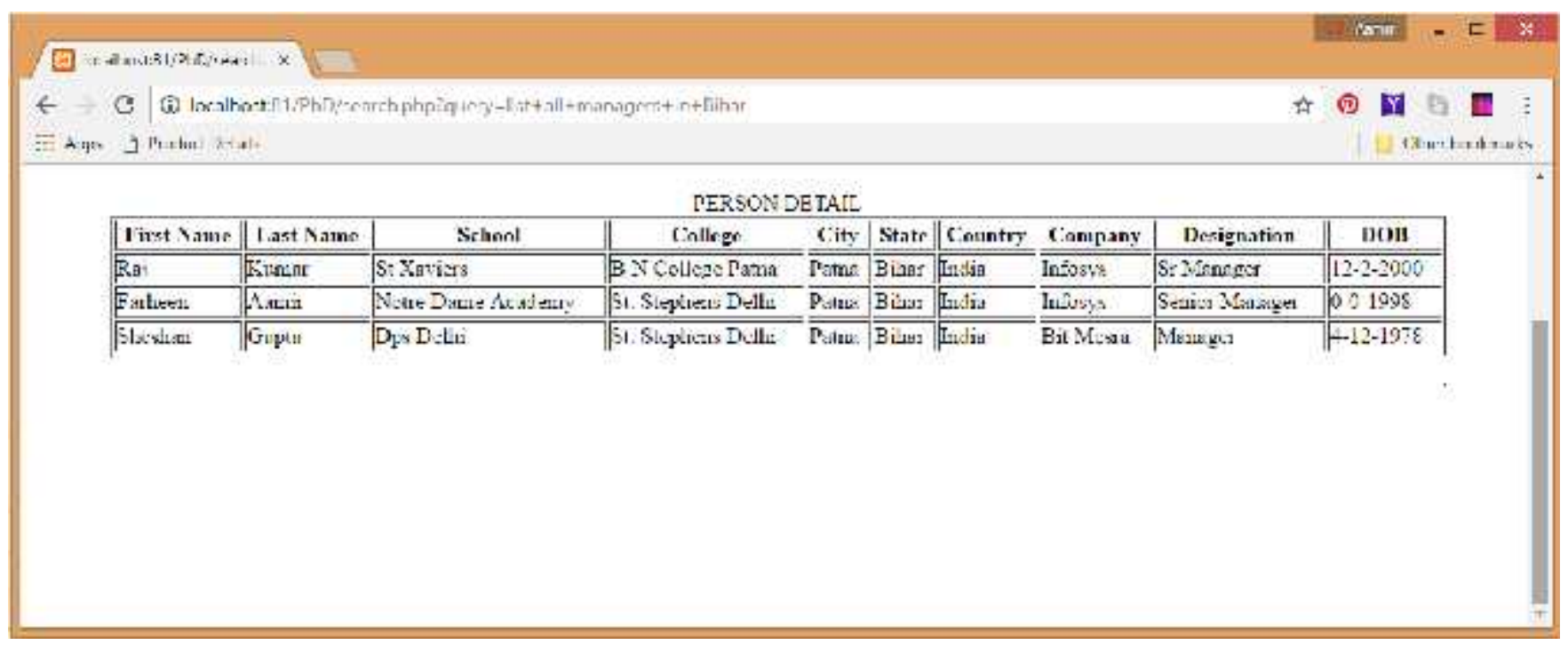

Figure 2 Output 


\section{CONCLUSION AND FUTURE WORK}

Finally we can say that the time complexity for the above algorithm is of the order $\mathrm{O}(\mathrm{n})$. In future we will try to increase the number of columns in the table Table1 to add more attributes which are used in user Profiles.

\section{Acknowledgment}

The authors would like to thank all faculty and students of Maulana Azad College of Engineering \& Technology, Patna who have shown interest and supported in this research. We are also very much thankful to our family members who have supported and cooperated us in this research work.

\section{References}

1. Aamir Junaid Ahmad and Sabina Priyadarshini. International Journal of Engineering Research \& Technology (IJERT) Vol. 3 Issue 9, September- 2014
2. Peter Mika, Social Network and the Semantic Web, http://www.springer.com/in/book/9780387710006

3. M. Jose A. Zarate1, R. Rodolfo A. Pazos1, Alexander Gelbukh2, and O Improving the Customization of Natural Language Interface to Databases Using an Ontology

4. list of citites in the world. http://brilliantmaps.com/ 4037-100000-person-cities/ 01/Jan/2017

5. list of countries in the world. http://www.infoplease. com/ ipa/ A0932875. html 01/Jan/2017

6. list of colleges and universities in the world http://www.4icu.org/reviews/index0001.htm01/Jan/ 2017

7. https://code.facebook.com/

\section{How to cite this article:}

Aamir Junaid Ahmad and Sabina Priyadarshini (2017) ' Searching A Social Networking Site Using Natural Language processing technique', International Journal of Current Advanced Research, 06(03), pp. 2473-2476.

DOI: http://dx.doi.org/10.24327/ijcar.2017.2476.0031 